\title{
The application of biomarkers of spermatogonial stem cells for restoring male fertility
}

\author{
Kevin T. Ebata, Jonathan R. Yeh, Xiangfan Zhang and Makoto C. Nagano* \\ Department of Obstetrics and Gynecology, McGill University, Royal Victoria Hospital, 687 Pine Avenue West, \\ Montreal, Quebec H3A 1A1, Canada
}

\begin{abstract}
Spermatogonial stem cells (SSCs) are defined by their ability to both self-renew and produce differentiated germ cells that will develop into functional spermatozoa. Because of this ability, SSCs can reestablish spermatogenesis after testicular damage caused by cytotoxic agents or after transplantation into an infertile recipient. Therefore, SSCs are an important target cell for restoring male fertility, particularly for cancer patients who have to undergo sterilizing cancer therapies. In the mouse, the identification of SSC markers allows for the isolation of a highly enriched population of stem cells. This enriched stem cell population can be expanded in culture for an indefinite period of time, cryopreserved, and transplanted into infertile recipients to restore fertility. Thus, the identification of markers and the establishment of a long-term culture system for human SSCs will be crucial for realizing the potential of these cells in a clinical setting. In this article, we focus on the markers that have been identified for mouse SSCs and discuss how human SSC markers may be used in the restoration of fertility.
\end{abstract}

Keywords: Spermatogenesis, stem cells, transplantation, cancer, male fertility restoration

\section{Stem cells in spermatogenesis}

Spermatogenesis is a complex and robust process by which numerous male gametes are continuously produced throughout life after puberty [20,31]. It is remarkable that an adult man is estimated to generate 1,000 sperm every heart beat [1], compared to one egg produced every 28 days in female gametogenesis. Spermatogenesis can be divided into three phases [52]. The first is the diploid phase that occurs on the basal membrane of the seminiferous epithelium, and is characterized by the proliferation of diploid germ cells, spermatogonia. Spermatogonia proliferate and expand the diploid germ cell population, but simultaneously undergo differentiation towards spermatozoa. In the second phase, the meiotic phase, differentiated spermatogonia leave the basal membrane and become spermatocytes

*Corresponding author: Fax: +1 514843 1662; E-mail: makoto. nagano@muhc.mcgill.ca. that undergo meiosis. Genetic recombination takes place in this phase. Resulting haploid cells are called spermatids. In the third phase, spermiogenesis, spermatids undergo complex morphological differentiation to form spermatozoa. These processes are supported and regulated by intimate interaction with somatic cells (Sertoli cells) in the seminiferous epithelium and by endocrine communication [20,31].

\subsection{Spermatogonial stem cells are defined by their regenerative activity}

At the foundation of spermatogenesis lie spermatogonial stem cells (SSCs). These cells are defined by their function: 1) to reproduce themselves (selfrenewal); 2) to generate progenitor spermatogonia that are committed to differentiation; and 3) to conduct the dual functions throughout life [5]. SSCs are a small fraction of spermatogonia, and represent only $0.01 \%$ of total testis cells in adult mice [40]. Compared to other 
A

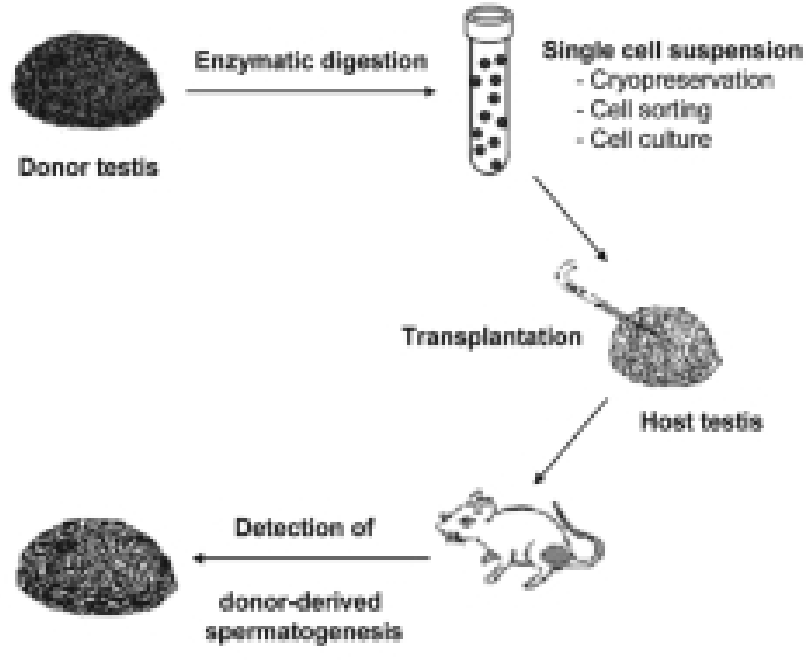

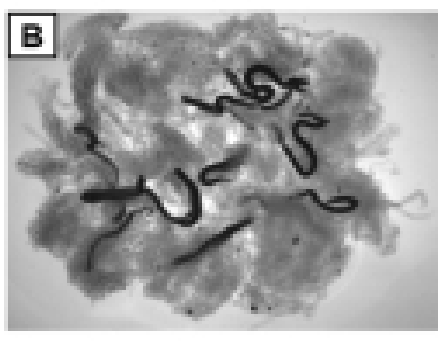

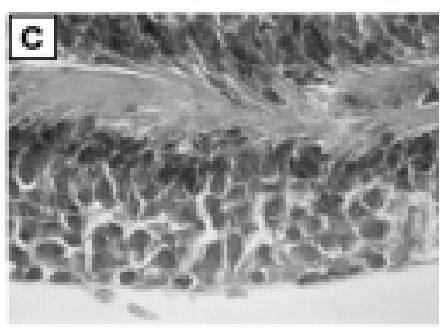

Fig. 1. Spermatogonial transplantation. (A) A schematic presentation of the transplantation procedure. (B) A wild-type host testis stained for LacZ activity 2 months after transplantation with donor transgenic mouse testis cells carrying LacZ. Note that donor-derived spermatogenesis can be observed as distinctive segments ("colonies", dark segments on the panel) along the seminiferous tubules. (C) A photomicrograph of histology of a colony. Spermatozoa are present, indicating the regeneration of complete spermatogenesis.

stem cell types, SSCs represent a unique stem cell population; they are the only cell type in adult mammals that can continuously self-renew and are responsible for transmitting appropriate genetic information to future generations. Furthermore, SSCs are critical for the continuation and evolution of the species, yet dispensable for the survival of an individual - the presence of germ cells does not significantly affect the longevity and the health of an individual. Another unique characteristic of SSCs is that a cell population equivalent to SSCs does not exist in the female germ line. In females, germ cells enter meiosis before birth and therefore, lose self-renewing potential. Thus, postnatal germ line stem cells exist only in the male.

\subsection{Clinical evidence implying the presence of SSCs in humans}

The presence of SSCs in humans is evidenced by the fact that a spontaneous restoration of male fertility can occur after potentially sterilizing anti-cancer therapy. For example, examining gonadal function in 53 men with Hodgkin's disease after two different regimens, Viviani et al. [58] found that 54\% of patients who received ABVD (doxorubicin, bleomycin, vincristine, and dacarbazine) experienced oligospermia. In patients who received MOPP (mechloethamine, vincristine, procarbazine, and prednisone), azoospermia occurred in $97 \%$ of the cases. Nevertheless, spermatogenesis recovery was observed in all patients who showed spermatogenic defects after the ABVD treatment and in $14 \%$ after the MOPP regimen. Since stem cells are defined by their ability to regenerate and maintain adult tissues [59], the recovery of spermatogenesis after anti-cancer treatment indicates the presence of SSCs.

\subsection{Spermatogonial transplantation - A functional assay of SSCs}

Due to their functional definition, stem cells are detected based on their regenerative activity. The functional assay of SSCs is spermatogonial transplantation, which is the only unequivocal SSC detection system available at present [5]. In this technique, a single-cell suspension of testis cells is injected into the seminiferous tubules of infertile hosts (Fig. 1). Upon injection, only SSCs regenerate and maintain donor-derived spermatogenesis, since injected donor germ cells at more advanced stages do not have a robust self-renewing capacity, and thus, will eventually disappear through differentiation or cell death. Supported by SSCs, donor spermatogenesis continues in the host testes, leading to fertility restoration; the hosts can sire offspring carrying donor-genotype $[6,44]$. Therefore, spermatogonial transplantation allows us to identify SSCs using their function as a marker. Although this technique was established and has been used most frequently in the study of mouse SSCs, it provides a potentially power- 

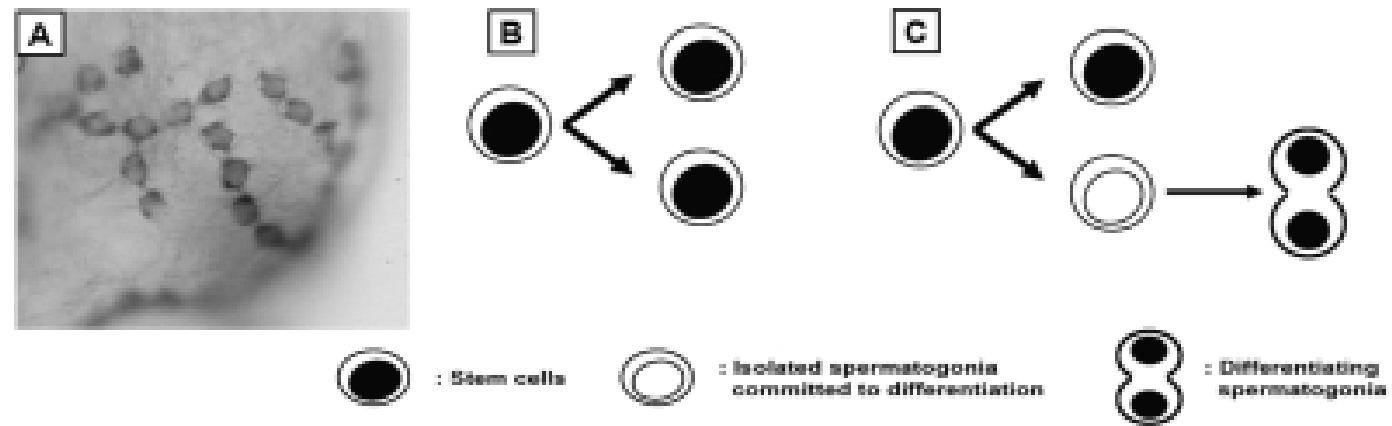

Fig. 2. (A) Chains of spermatogonia. Incomplete cytokinesis of male germ cells results in the formation of chains of cells that are connected by cytoplasmic bridges. (B) and (C) Two patterns of SSC fate after a division. SSCs are believed to be a subpopulation of isolated single spermatogonia. Upon a division, one SSC can produce either two SSCs (B), or one SSC and one isolated single spermatogonium (C). In the latter case, one isolated spermatogonium generates a chain of two cells at the next division, and thus, it loses self-renewal activity; i.e., this cell is retrospectively determined to be a committed progenitor, but not a stem cell, despite its identity as an isolated spermatogonium.

ful approach to restoring male fertility in humans, as described in the last section of this article.

Spermatogonial transplantation also allows quantification of SSCs. Upon transplantation, donor-derived spermatogenesis is reconstituted in the form of distinctive segments along the seminiferous tubules [43] (Fig. 1). Recent studies have demonstrated that each of these segments (termed "colonies") arises from one single stem cell $[27,63]$. Therefore, by simply counting the number of colonies, SSCs can be quantified reliably. The quantitative nature of the transplantation assay has been critical to determine SSC identification markers.

In this article, various biomarkers of SSCs will be first described, followed by the potential application of these markers in a SSC-based male fertility restoration scheme. As our knowledge about human SSCs is highly limited, most discussion is based on animal studies using the mouse as a model species.

\section{Biomarkers of spermatogonial stem cells}

In general, SSC markers can be categorized into three different types: morphological, cell-surface, and intracellular markers (Table 1).

\subsection{Morphological markers}

The morphological identification of SSCs, both in animal models and humans, relies on a unique characteristic of male germ cell division; their cytokinesis is incomplete, and the majority of male germ cells are associated through a cytoplasmic bridge after division (Fig. 2). Thus, differentiating germ cells from sper-
Table 1

Mouse SSC markers

\begin{tabular}{ll}
\hline Positive markers & Negative markers \\
\hline Cell-surface & \\
Integrin $\alpha 6$ & Integrin $\alpha \mathrm{v}$ \\
Integrin $\beta 1$ & c-kit \\
Thy-1 & MHC-I \\
CD9 & CD34 \\
CD24 & \\
GFR $\alpha 1$ & \\
Side population & \\
Hoechst 33342 efflux & \\
Rhodamine 123 efflux & \\
Intracellular markers & \\
Oct4 & \\
Plzf & \\
Neurogenin3 & \\
Stra8 & \\
\hline
\end{tabular}

matogonia to spermatid stages synchronously develop as a clone [4,52]. The only exception is seen at the very early stage of spermatogonia. Some primitive spermatogonia exist as isolated single cells, without association with other germ cells through the cytoplasmic bridge. Upon division, these single cells either complete the cytokinesis and produce two isolated spermatogonia, or undergo incomplete cytokinesis and generate chains of two spermatogonia [39] (Fig. 2). Since the formation of a two-cell chain is believed to be the first step towards terminal spermatogenic differentiation, the isolated spermatogonia are often called stem cells, because of their potential to self-renew (producing two single cells) or differentiate (producing a twocell chain). However, a caution is necessary. As noted above, stem cells are defined by their function and "retrospectively" detected by spermatogonial transplantation. In contrast, this morphological characteristic is used as a prospective marker, with no functional rela- 
tion to how cells will behave at the next cell division (Fig. 2). Therefore, it is reasonable to recognize that even though stem cells may well be isolated spermatogonia, the inverse may not be true; i.e., isolated spermatogonia are not necessarily stem cells, since they may lose self-renewing ability at the next cell division by producing paired cells that are committed to terminal differentiation.

\subsection{Cell-surface markers}

In contrast to morphological markers, cell-surface molecules have been determined as SSC markers by directly associating their expression with stem cell activity. Integrin $\alpha 6$ and $\beta 1$ were the first cell-surface markers identified [55], which together form a receptor of laminin, a major component of extracellular matrix in the basal membrane of the seminiferous epithelium. Mouse testis cells can be immunologically sorted, using a magnet-activated cell sorting (MACS) technique, based on the expression of integrin $\alpha 6$ or $\beta 1$. When transplanted into host testes, integrin $\alpha 6+$ or $\beta 1+$ cells produce a significantly greater number of spermatogenic colonies than unsorted cells, indicating that SSCs are enriched in the integrin $\alpha 6+$ or $\beta 1+$ cell population [55]. Using similar immunological approaches combined with spermatogonial transplantation, Thy-1 (CD90), CD9, and CD24 have been identified as the molecules expressed on SSCs [30,33]. On the other hand, integrin $\alpha \mathrm{v}$, c-kit, Sca-1, CD34, and MHC-I are negative markers as they do not allow SSC enrichment $[33,55]$.

Using fluorescent-activated cell sorting (FACS), mouse SSCs can now be enriched up to 700-fold in the Thy- ${ }^{+} \mathrm{MHC}^{-}{ }^{-} \mathrm{Kit}^{-}$fraction [33]. However, it should be noted that a large degree of SSC enrichment is achieved prior to immunological cell sorting by experimental cryptorchidism. This testis condition is induced by suturing testes to the abdominal wall, resulting in a cessation of spermatogenesis due to exposure to a high body core temperature. Since SSCs survive the cryptorchid testis environment, they are enriched 20to 25-fold [40,56]. Thus, the contribution of immunological cell sorting to SSC enrichment is estimated to be as much as $\sim 35$-fold (700/20). Since the frequency (density) of SSCs in an adult mouse testis is estimated to be $0.01 \%[39,40]$, the highest SSC enrichment of 700 -fold may provide a cell population that contains one SSC in 14 sorted cells (7\%).

Cell-surface molecules are ideal SSC markers as their identity is directly linked to regenerative ability that allows us to select functional live cells. However, they are not without limitations. Most critically, these molecules are not exclusively expressed by SSCs. For example, Thy-1 is a well-known lymphocyte marker, and integrin $\beta 1$ is expressed also in Sertoli cells [25, 60]. Thus, as seen with morphological markers, a given cell-surface molecule may be expressed by SSCs, but a single marker can not uniquely identify SSCs. Further efforts are necessary to determine the combination of cell-surface markers that can lead to SSC purification (i.e., $100 \%$ enrichment).

Another SSC cell-surface marker is GFR $\alpha 1$, a receptor of glial cell line-derived neurotrophic factor (GDNF) [9,12]. GDNF is an essential growth factor for SSC maintenance and promotes a robust SSC expansion in vitro [28,34]. Interestingly, the expression of GFR $\alpha 1$ appears to be age-dependent. When one-weekold mouse testis cells were sorted and transplanted, a significant SSC enrichment was detected in GFR $\alpha 1+$ cells $[9,12]$. However, SSCs were not enriched in GFR $\alpha 1+$ cells when adult or new born mouse testis cells were used as donors [12]. Therefore, it appears that GFR $\alpha 1$ expression may be elevated after birth, but decline by adulthood in mice. It may be possible that some markers are expressed differentially depending on age or other physiological parameters, such as cell cycle stages.

\subsection{Intracellular markers}

Recent mouse studies have also indicated that SSCs express intracellular molecules, such as transcription factors (Oct4, Plzf, and neurogenin3) and a cytoplasmic protein (Stra8) $[8,15,19,47,62]$. Similar to other markers described above, these molecules are not exclusively expressed in SSCs, but expressed also in primitive, but differentiating, spermatogonia. In addition, since these molecules are expressed inside the cell, live SSCs cannot be sorted based on the expression of intracellular markers using immunological methods, unless the cells are engineered for labeling with these molecules (e.g., Oct4-GFP transgenic reporter line). However, these marker molecules may well be involved in the regulatory mechanism of SSC proliferation, differentiation, and survival. Therefore, the identification of these molecules provides opportunities to further explore the functional characterization of SSCs. 


\subsection{Side population}

In addition to the three types of markers described above, various types of stem cell populations (e.g., hematopoietic and neural stem cells) have also been enriched on the basis of their activity to efflux Hoechst DNA-binding dyes [16,23]. Due in part to the presence of ATP-binding cassette transporters (MDR1, BR$\mathrm{CP} 1 / \mathrm{ABCG} 2)$ on the cell surface, cells can efflux the Hoechst dye, and those that are selected using flow cytometry for less intensive dye-staining include a high proportion of stem cells: this cell population is termed "side population" (SP) [16,23]. In the testis, conflicting data have been reported as to if SSCs are enriched in the SP. Kubota et al. [33] initially reported the absence of SSCs in the testis SP using donor cryptorchid testis cells. However, two studies later obtained $\mathrm{a} \sim 15$ fold enrichment of SSCs in the SP using intact testis cells $[13,36]$. The discrepancy between the studies was postulated to be due to the condition of donor testes (cryptorchid vs. intact testes); SSCs derived from cryptorchid testes were suggested to be more severely affected by the toxicity of the dye [38]. More recently, using a less toxic dye, rhodamine 123 (a mitochondrial dye), Lo et al. [38] obtained a SP from donor cryptorchid testis cells that was enriched $\sim 20$-fold for SSC activity. This suggests that the ability to efflux these dyes is a characteristic of SSCs regardless of donor testis condition, and may be used in combination with cell surface markers to further enrich for stem cell activity. As the isolation of hematopoietic stem cells (HSCs) using the SP has been achieved in multiple species [17], including humans, it is possible that the SSC SP phenotype may also be conserved across species. Since the cell surface phenotype of human SSCs is currently unknown, the detection of a SP in human testis cells may be an effective method for isolating human SSCs without knowing their cell surface phenotype [36].

\subsection{Cell-surface markers, SSC enrichment, and in vitro SSC culture}

Among the three types of SSC markers described above, cell-surface markers provide the best established means to obtain live SSCs. Recently, an in vitro system was developed, in which mouse SSCs can be maintained for a long time and amplified robustly [28,29, 34]. In this system, mouse testis cells are first enriched for SSCs, and placed on a feeder layer of embryonic mouse fibroblasts. Then, the SSC-enriched cells are cultured in serum-free or -reduced medium supplemented with GDNF and FGF2. Within 6 days in vitro, these cells form "clusters" of spermatogonia (Fig. 3) [28,34]. Transplantation of clusters results in a complete regeneration of spermatogenesis in host mouse testes, demonstrating that clusters contain SSCs. Serial passaging of clusters results not only in a longterm (at least 2 years) maintenance of SSCs but also in a robust expansion of SSCs in vitro (1,000-fold expansion in 40 days. Fig. 3) [28,34]. Importantly, cluster formation and SSC expansion cannot be achieved without an enrichment procedure, since testicular somatic cells overwhelm the SSC culture.

This culture system reflects two of the stem cell definition parameters, i.e., self-renewal and long-term activity. However, the differentiation activity is not represented in the culture system, due simply to a technical limitation: reconstruction of complete spermatogenesis is not yet feasible in vitro. Despite this limitation, the culture system now provides the opportunity to investigate SSC regulatory mechanisms under a defined condition, and to expand the SSC population. Since SSCs represent an extremely small proportion of testis cells $[39,40]$, amplification of SSCs allows us to obtain a sufficient quantity for experimentation, and potentially for efficient male fertility restoration in clinical settings in the future.

\subsection{Human SSC markers}

Studies of human spermatogonia have relied largely on cell morphology [39], and our knowledge about human SSCs and their markers is highly limited. It is believed, however, that human SSCs are a subpopulation of isolated spermatogonia [39]. The progress of SSC studies in the mouse model should provide a foundation for studies of human SSCs and their markers. For example, since the extracellular matrix components of basal membrane are similar in mouse and human testes, integrin molecules may be cell-surface markers for human SSCs [37,53]. Thus, the molecules expressed by mouse SSCs will be the primary candidates in the efforts to identify human SSC markers.

A significant issue in studying human SSCs is that no functional assay is available at present. Although human spermatogonia survive for at least 6 months and show some proliferation in the testes of immunodeficient mice after transplantation, germ cell differentiation does not proceed and human spermatogenesis cannot be regenerated in mouse testes [45]. Nonetheless, transplantation of human germ cells into mouse testes may still be the best option currently available 

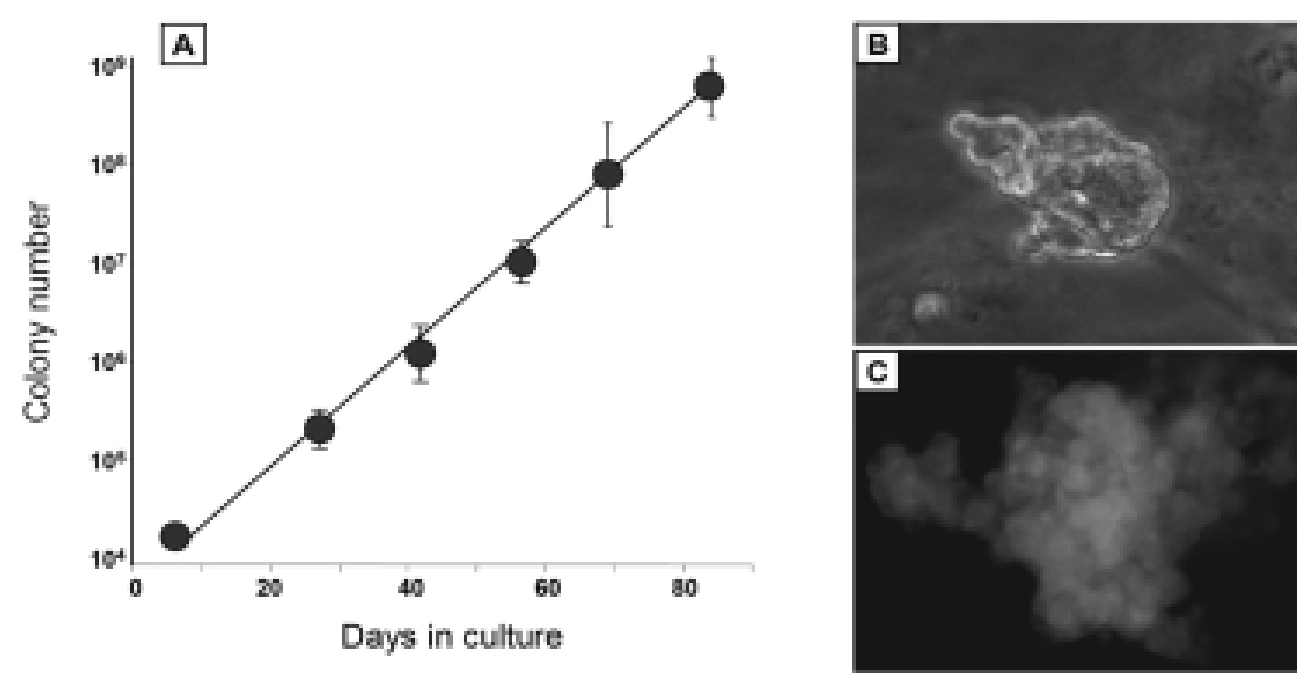

Fig. 3. (A) SSCs can be cultured for a long time. The results of periodic transplantation of cultured cells demonstrate that SSCs robustly expand in vitro, as indicated by the increase in colony numbers after transplantation. (B) In this culture system, SSCs form aggregates of spermatogenic cells, termed "clusters". (C) The cluster cells show the alkaline phosphatase activity, although it is not detected in postnatal male germ cells in vivo. From our unpublished data [61].

for identifying human SSC markers. Among various types of human testis cells, only spermatogonia survive in mouse testes for a long time after transplantation, and some even show proliferation activity, generating small groups of germ cells [45]. Thus, following immunological cell sorting and transplantation, the number of long-term surviving spermatogonia and the number of germ cell groups in host mouse testes could be used as a quantitative index of enrichment efficiency for human primitive spermatogonia that can colonize mouse testes. Then, potential human SSC markers can be identified as done with mouse SSC markers.

Another approach is to use the SSC culture system for SSC marker identification. Since the number of germ cell clusters produced in vitro faithfully reflects the number of SSCs [61], SSC enrichment could be measured by counting the number of clusters: i.e., if a given cell-surface molecule is expressed by SSCs, then, cells selected for such a molecule should produce more clusters. Although human SSC culture has not been established, it should be an effective alternative approach to identifying human SSC markers by replacing the transplantation assay.

\subsection{SSCs and germ cell tumors}

In relation to SSC culture, it may be worthwhile to discuss a possibility that SSCs may be involved in germ cell tumor formation. Germ cell tumors (e.g., seminomas, teratomas) arise from testicular carcinoma-in- situ (CIS) [50,51,57]. CIS cells are identified based on their alkaline phosphatase (AP) activity in histological sections. A wealth of evidence indicates that human fetal gonocytes are very similar to CIS cells and may be their origin [50]. For example, there is a close morphological similarity between CIS cells and human fetal gonocytes. Also, CIS cells express, in addition to AP, an array of marker molecules expressed by fetal gonocytes, such as c-kit and Oct4. Furthermore, germ cell tumors are more frequently seen early in life, and their occurrence declines in old age [50]. However, recent in vitro studies of mouse SSCs have presented interesting observations that may suggest a linkage of postnatal SSCs to germ cell tumors.

As seen in humans, postnatal germ cells in mice do not show the AP activity. Interestingly, however, cells in germ cell clusters derived from postnatal mouse SSCs in vitro express this enzyme [34] (Fig. 3). Clusters also express Oct4 and c-kit, as they are formed by SSCs and spermatogonia [28,34]. In addition, there is evidence that cluster cells can transform to embryonic stem cells (ESCs), the stem cell of teratocarcinomas: this remarkable transformation is facilitated by a mutation in p53, an anti-oncogenic gene [26]. Recent studies further indicate that SSCs during early postnatal development have different functional properties than adult SSCs, as immature SSCs divide more actively than adult SSCs $[11,40,46]$. Although still circumstantial, these observations collectively suggest a possibility that not only fetal gonocytes but also postnatal SSCs, 
which may have taken an aberrant developmental path, could be the origin of CIS and germ cell tumors.

\section{Application of human spermatogonial stem cells in male fertility restoration, and needs for future research development}

Potential applications of SSCs have been discussed in various articles, including male fertility restoration, germ-line gene therapy, production of human ESCs, transgenesis in farm animals, and conservation of endangered species $[6,7,21,35,41,42]$. Here, we focus on the use of SSC markers in stem cell-based male fertility restoration.

As the cancer survival rate increases, sterilizing chemo- and radiation therapies have become a significant concern for the quality of life of cancer survivors [22]. Spermatogenesis is highly sensitive to cancer therapy, causing more infertility in men than in women [10,54]. Although semen cryopreservation is currently the only option to preserve male fertility, the quality and quantity of sperm are often not optimal in cancer patients, and the success rate of this approach can be as low as $25 \%$ [3]. Furthermore, this concern is particularly serious for childhood cancer patients, since semen cryopreservation is not an option for them to have genetic children [18,22]. However, as SSCs are consistently present in the testis after birth, this problem may be overcome by spermatogonial transplantation.

A general procedure of this approach is to first harvest a testis biopsy from a patient before cancer therapy, cryopreserve germ cells including SSCs, and transplant them back to the patient after the cytotoxic effect of anti-cancer agents has subsided (Fig. 4). It has been shown that SSC cryopreservation is possible in many species, using a simple freezing medium developed for somatic cells $[2,21,45]$. Since SSCs are diploid cells, cryopreservation protocols used for somatic cells appear to be readily applicable to SSCs across species.

This transplantation scheme for male fertility restoration has been applied in a clinical trial by Redford et al. in 1999, and the results should appear in the near future $[48,49]$. The caveat of this trial is that it would be difficult to determine if spermatogenic regeneration was achieved by transplanted SSCs or by endogenous SSCs that survived anti-cancer therapy. On the other hand, it may also be possible that transplantation of SSCs that have not experienced the cytotoxic effect of anti-cancer agents may ameliorate the testis environment and encourage the regenerative activity of surviving endogenous SSCs, leading to more efficient spermatogenic regeneration than with surviving SSCs alone.

Two interrelated, critical issues need to be considered to realize this SSC-based male fertility restoration scheme. First, since SSCs are harvested prior to cancer therapy, tumorigenic cells may be included in a biopsy, presenting a risk of cancer relapse. As germ cells are dispensable for the life of cancer survivors, this is obviously not acceptable in clinical settings. Second, a biopsy specimen that can be harvested from cancer patients, particularly pediatric cancer patients, may well be small in size. Considering the rarity of SSCs in total testis cells, the quantity of SSCs in such a testis biopsy may be a limiting factor for efficient fertility restoration. Although technologies need to be established for human SSCs, studies using animal models have indicated that these problems can potentially be overcome in clinical situations by effective applications of SSC markers.

Using a rat model, Jahnukainen and colleagues showed that T-cell leukemia can recur upon transplantation of contaminated testis cells [24]. They estimated that as few as 20 leukemic cells can cause a cancer relapse. To eliminate such a risk, SSCs need to be purified, and/or tumor cells purged using cell-surface markers; the former approach represents a positive selection, and the latter a negative selection. Although the purification of SSCs appears ideal, it has not been achieved even in the mouse model. In addition, the positive selection scheme requires a premise that SSCs and cancer cells do not share a common cell-surface marker. For examples, Thy- 1 is currently one of the markers that allows effective enrichment of SSCs in mice, but it is expressed in the T-cell lineage and HSCs [59], rendering separation of SSCs from leukemic cells difficult.

In contrast, it may be more practical to purge tumor cells from a testis cell population, even if it does not allow purification of SSCs. Here, we can take an advantage of a unique SSC characteristic. As described above, spermatogonia, including SSCs, do not express MHC-I molecules in mice as well as in humans [32,33]; thus, cell sorting using this SSC negative marker will be a powerful approach to purge tumor cells with minimal stem cell loss. In the case of hematological cancers, SSCs do not express a pan-hematopoietic marker, CD45 [14] (our unpublished data), at least in mice, which can be used to negatively sort out cells in the hematopoietic lineage. In fact, using the mouse model, Fujita and co-workers have recently shown that this strategy is effective in male fertility restoration [14]. 


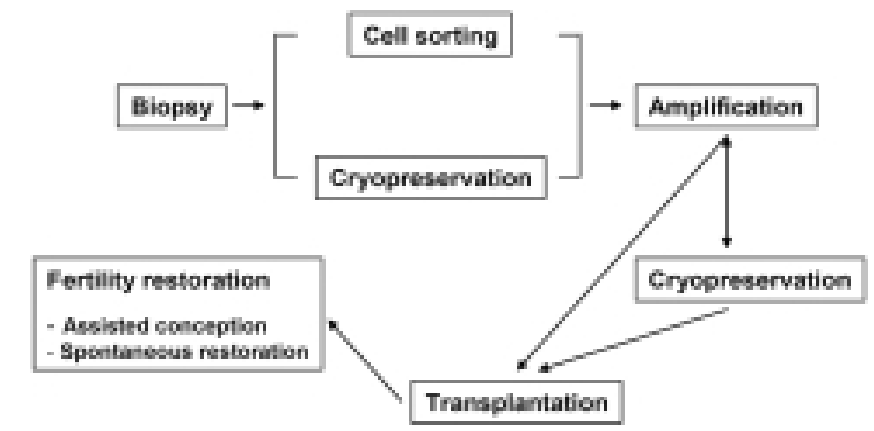

Fig. 4. A potential scheme of SSC-based male fertility restoration. A biopsy specimen is enzymatically digested to produce a single cell suspension. This preparation can be cryopreserved or used for cell sorting to isolate SSCs or purge tumorigenic cells. Next, SSCs are amplified in vitro, which can be cryopreseved for future use or transplanted back to a patient. Cryopreseved cells can be transplanted or re-amplified after thawing. Transplantation of SSCs is expected to lead to spontaneous fertility recovery, or provide opportunities for assisted reproduction.

They used FACS to eliminate $\mathrm{H}-2 \mathrm{~K}^{\mathrm{b}} / \mathrm{H}-2 \mathrm{D}^{\mathrm{b}}$ (MHC class I heavy chain) -positive and CD45-positive cells from testis cells of mice inoculated with leukemic cell lines. The negatively sorted cells were enriched for germ cells, and following transplantation, regenerated spermatogenesis without the relapse of leukemia. Furthermore, normal progeny were produced by intracytoplasmic injection of sperm harvested from the host mouse testes, demonstrating the effectiveness of the negative selection strategy.

The second critical issue is the quantity of SSCs. Since a testis biopsy is expected to be small in size, sorted cells may contain only a small number of SSCs following tumor cell purging. In addition, cryopreservation may reduce the number of viable SSCs for transplantation. To circumvent these expected problems, an in vitro culture system needs to be developed for human SSCs. As seen with mouse SSCs, it should be possible to amplify human SSCs in vitro and obtain a large number of them. With sufficient SSCs in hand, repeated transplantation may be effective to restore fertility. The abundance of SSCs available will also allow us to confirm the absence of tumorigenic cells in the cell preparation. This could be done with cytological approaches (morphology, cell staining using lineagespecific markers) or in culture (transferring cultured testicular cells into an optimal culture condition for a given cancer cell type, and detecting the presence or absence of proliferating tumorigenic cells). Thus, the establishment of a human SSC culture technique is a critical target for future research.

\section{Conclusions}

Although our knowledge about human SSCs is currently limited, recent developments in SSC research using animal models has rapidly provided sufficient information and a technical basis to extend our research to human SSCs. Two critical research areas should be the identification of human SSC markers, particularly cell-surface markers, and the establishment of a human SSC culture system. As mouse SSC culture systems require SSC enrichment, these two issues are interrelated, and studies to address them are likely to proceed simultaneously. Certainly, such investigations must be conducted with careful consideration of ethical issues, especially concerning the experimental use of testicular biopsies derived from prepubertal boys. Nevertheless, our ability to enrich and culture SSCs using their markers should have a significant impact on improving the quality of life of cancer survivors in the near future.

\section{References}

[1] R.P. Amann and S.S. Howards, Daily spermatozoal production and epididymal spermatozoal reserves of the human male, $J$ Urol 124(2) (1980), 211-215.

[2] M.R. Avarbock, C.J. Brinster and R.L. Brinster, Reconstitution of spermatogenesis from frozen spermatogonial stem cells, Nat Med 2(6) (1996), 693-696.

[3] F.H. Blackhall et al., Semen cryopreservation, utilisation and reproductive outcome in men treated for Hodgkin's disease, Br J Cancer 87(4) (2002), 381-384.

[4] R.E. Braun et al., Genetically haploid spermatids are phenotypically diploid, Nature 337(6205) (1989), 373-376.

[5] R.L. Brinster, Germline stem cell transplantation and transgenesis, Science 296(5576) (2002), 2174-2176.

[6] R.L. Brinster and M.R. Avarbock, Germline transmission of donor haplotype following spermatogonial transplantation, Proc Natl Acad Sci USA 91(24) (1994), 11303-11307.

[7] R.L. Brinster and J.W. Zimmermann, Spermatogenesis following male germ cell transplantation, Proc Natl Acad Sci USA 91(24) (1994), 11298-11302.

[8] F.W. Buaas et al., Plzf is required in adult male germ cells for stem cell self-renewal, Nat Genet 36(6) (2004), 647-652. 
[9] A. Buageaw et al., GDNF family receptor alpha1 phenotype of spermatogonial stem cells in immature mouse testes, Biol Reprod 73(5) (2005), 1011-1016.

[10] R.M. Chapman, S.B. Sutcliffe and J.S. Malpas, Cytotoxicinduced ovarian failure in women with Hodgkin's disease, JAMA 242(17) (1979), 1877-1886.

[11] K.T. Ebata, X. Zhang and M.C. Nagano, Male germ line stem cells have an altered potential to proliferate and differentiate during postnatal development, Biol Reprod, E-pub on 17 January, 2007: 10.1095/biolreprod.106.058305.

[12] K.T. Ebata, X. Zhang and M.C. Nagano, Expression patterns of cell-surface molecules on male germ line stem cells during postnatal mouse development, Mol Reprod Dev 72(2) (2005), 171-181.

[13] I. Falciatori et al., Identification and enrichment of spermatogonial stem cells displaying side-population phenotype in immature mouse testis, FASEB J 18(2) (2004), 376-378.

[14] K. Fujita et al., Transplantation of spermatogonial stem cells isolated from leukemic mice restores fertility without inducing leukemia, J Clin Invest 115(7) (2005), 1855-1861.

[15] G. Giuili et al., Murine spermatogonial stem cells: targeted transgene expression and purification in an active state, $E M B O$ Rep 3(8) (2002), 753-759.

[16] M.A. Goodell et al., Isolation and functional properties of murine hematopoietic stem cells that are replicating in vivo, $J$ Exp Med 183(4) (1996), 1797-1806.

[17] M.A. Goodell et al., Dye efflux studies suggest that hematopoietic stem cells expressing low or undetectable levels of CD34 antigen exist in multiple species, Nat Med 3(12) (1997), 13371345.

[18] R.G. Gosden et al., Gonadal tissue cryopreservation: clinical objectives and practical prospects, Hum Fertil 2(2) (1999), $107-114$.

[19] J.A. Gostoya et al., Essential role of Plzf in maintenance of spermatogonial stem cells, Nat Genet 36(6) (2004), 653-659.

[20] M.D. Griswold, Interactions between germ cells and Sertoli cells in the testis, Biol Reprod 52(2) (1995), 211-216.

[21] J.R. Hill and I. Dobrinski, Male germ cell transplantation in livestock, Reprod Fertil Dev 18(1-2) (2006), 13-18.

[22] S. Howell and S. Shalet, Pharmacological protection of male fertility, in: Preservation of Fertility, T. Tulandi and R.G. Gosden, eds, Taylor \& Francis, London, 2004, pp. 77-82.

[23] R. Hulspas and P.J. Quesenberry, Characterization of neurosphere cell phenotypes by flow cytometry, Cytometry 40(3) (2000), 245-250.

[24] K. Jahnukainen et al., Intratesticular transplantation of testicular cells from leukemic rats causes transmission of leukemia, Cancer Res 61(2) (2001), 706-710.

[25] C.A. Janeway Jr, P. Travers, M. Walport and M.J. Shlomchik, Immunobiology: the immune system in health and disease, $5^{\text {th }}$ ed., Garland Publishing, New York, 2001, 666.

[26] M. Kanatsu-Shinohara et al., Generation of pluripotent stem cells from neonatal mouse testis, Cell 119(7) (2004), 10011012 .

[27] M. Kanatsu-Shinohara et al., Clonal origin of germ cell colonies after spermatogonial transplantation in mice, Biol Reprod 75(1) (2006), 68-74.

[28] M. Kanatsu-Shinohara et al., Long-term proliferation in culture and germline transmission of mouse male germline stem cells, Biol Reprod 69(2) (2003), 612-616.

[29] M. Kanatsu-Shinohara et al., Genetic and epigenetic properties of mouse male germline stem cells during long-term culture, Development 132(18) (2005), 4155-4163.
[30] M. Kanatsu-Shinohara, S. Toyokuni and T. Shinohara, CD9 is a surface marker on mouse and rat male germline stem cells, Biol Reprod 70(1) (2004), 70-75.

[31] A.L. Kierszenbaum, Mammalian spermatogenesis in vivo and in vitro: a partnership of spermatogenic and somatic cell lineages, Endocrine Reviews 15(1) (1994), 116-134.

[32] J. Klein, Natural History of Major Histocompatibility Complex, Wiley, New York, 1986, 152-175.

[33] H. Kubota, M.R. Avarbock and R.L. Brinster, Spermatogonial stem cells share some, but not all, phenotypic and functional characteristics with other stem cells, Proc Natl Acad Sci USA 100(11) (2003), 6487-6492.

[34] H. Kubota, M.R. Avarbock and R.L. Brinster, Growth factors essential for self-renewal and expansion of mouse spermatogonial stem cells, Proc Natl Acad Sci USA 101(47) (2004), 16489-16494.

[35] H. Kubota and R.L. Brinster, Technology insight: In vitro culture of spermatogonial stem cells and their potential therapeutic uses, Nat Clin Pract Endocrinol Metab 2(2) (2006), 99-108.

[36] B. Lassalle et al., 'Side population' cells in adult mouse testis express Bcrp1 gene and are enriched in spermatogonia and germinal stem cells, Development 131(2) (2004), 479-487.

[37] D.R. Lee et al., Isolation of male germ stem cell-like cells from testicular tissue of non-obstructive azoospermic patients and differentiation into haploid male germ cells in vitro, Hum Reprod 21(2) (2006), 471-476.

[38] K.C. Lo et al., Isolation and enrichment of murine spermatogonial stem cells using rhodamine 123 mitochondrial dye, Biol Reprod 72(3) (2005), 767-771.

[39] M.L. Meistrich and M.E.A.B. van Beek, Spermatogonial stem cells, in: Cell and molecular biology of the testis C. Desjardins and L.L. Ewing, eds, Oxford University Press, New York, 1993, pp. 266-295.

[40] M.C. Nagano, Homing efficiency and proliferation kinetics of male germ line stem cells following transplantation in mice, Biol Reprod 69(2) (2003), 701-707.

[41] M.C. Nagano, Spermatogonial Stem Cells, in: Pharmacological protection of male fertility, In: Preservation of Fertility, T. Tulandi and R.G. Gosden, eds, Taylor \& Francis, London, 2004, pp. 125-139.

[42] M.C. Nagano, Germ Line Stem Cells, in: Stem Cells in Endocrinology, L.B. Lester, ed., Humana Press, Totowa, 2005, pp. $23-47$.

[43] M. Nagano, M.R. Avarbock and R.L. Brinster, Pattern and kinetics of donor mouse spermatogonial stem cell colonization in recipient testes, Biol Reprod 60(6) (1999), 1429-1436.

[44] M. Nagano et al., Transgenic mice produced by retroviral transduction of male germ line stem cells, Proc Natl Acad Sci USA 98(23) (2001), 13090-13095.

[45] M. Nagano, P. Patrizio and R.L. Brinster, Long-term survival of human spermatogonial stem cells in mouse testes, Fertil Steril 78(6) (2002), 1225-1233.

[46] M. Nagano et al., Lentiviral vector transduction of male germ line stem cells in mice, FEBS Lett 524(1-3) (2002), 111-115.

[47] K. Ohbo et al., Identification and characterization of stem cells in prepubertal spermatogenesis in mice, Dev Biol 258(1) (2003), 209-225.

[48] J. Radford, Restoration of fertility after treatment for cancer, Horm Res 59(Suppl 1) (2003), 21-23.

[49] J. Radford, S. Shalet and B. Lieberman, Fertility after treatment for cancer. Questions remain over ways of preserving ovarian and testicular tissue, BMJ 319(7215) (1999), 935-936. 
[50] E. Rajpert-De Meyts, Developmental model for the pathogenesis of testicular carcinoma in situ: genetic and environmental aspects, Hum Reprod Update 12(3) (2006), 303-323.

[51] E. Rajpert-De Meyts et al., The emerging phenotype of the testicular carcinoma in situ germ cell, APMIS 111(1) (2003), 267-278.

[52] L.D. Russell, R.A. Ettlin, A.P. Sinha Hikim and E.D. Clegg, Hitological and Histopathological Evaluation of the Testis, Cache River Press, Clearwater, 1990, 1-58.

[53] J. Schaller, H.J. Glander and J. Dethloff, Evidence of beta 1 integrins and fibronectin on spermatogenic cells in human testis, Hum Reprod 8(11) (1993), 1873-1878.

[54] R.L. Schilsky et al., Gonadal dysfunction in patients receiving chemotherapy for cancer, Ann Intern Med 93(1) (1980),: 109114.

[55] T. Shinohara, M.R. Avarbock and R.L. Brinster, $\beta_{1^{-}}$and $\alpha_{6}{ }^{-}$ integrin are surface markers on mouse spermatogonial stem cells, Proc Natl Acad Sci USA 96(10) (1999), 5504-5509.

[56] T. Shinohara, M.R. Avarbock and R.L. Brinster, Functional analysis of spermatogonial stem cells in Steel and cryptorchid infertile mouse models, Dev Biol 220(2) (2000), 401-411.

[57] N.E. Skakkebaek, Possible carcinoma-in-situ of the testis, Lancet 2(7776) (1972), 516-517.
[58] S. Viviani, A. Santoro and G. Ragni, Gonadal toxicity after combination chemotherapy for Hodgkin's disease. Comparative results for MOPP vs ABVD, Eur J Cancer Clin Oncol 21(5) (1984), 601-605.

[59] I.L. Weissman, Translating stem and progenitor cell biology to the clinic: barriers and opportunities, Science 287(5457) (2000), 1442-1446.

[60] H.H. Yan and C.Y. Cheng, Laminin alpha 3 forms a complex with beta3 and gamma3 chains that serves as the ligand for alpha 6beta1-integrin at the apical ectoplasmic specialization in adult rat testes, J Biol Chem 281(25) (2006), 17286-17303.

[61] J.R. Yeh, X. Zhang and M.C. Nagano, Establishment of a short-term in vitro assay for mouse spermatogonial stem cells, Biol Reprod 77 (2007), in press.

[62] S. Yoshida et al., Neurogenin3 delineates the earliest stages of spermatogenesis in the mouse testis, Dev Biol 269(2) (2004), $447-458$

[63] X. Zhang, K.T. Ebata and M.C. Nagano, Genetic analysis of the clonal origin of regenerating mouse spermatogenesis following transplantation, Biol Reprod 69(6) (2003), 18721878. 


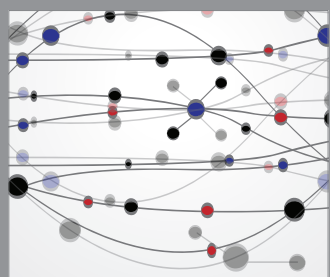

The Scientific World Journal
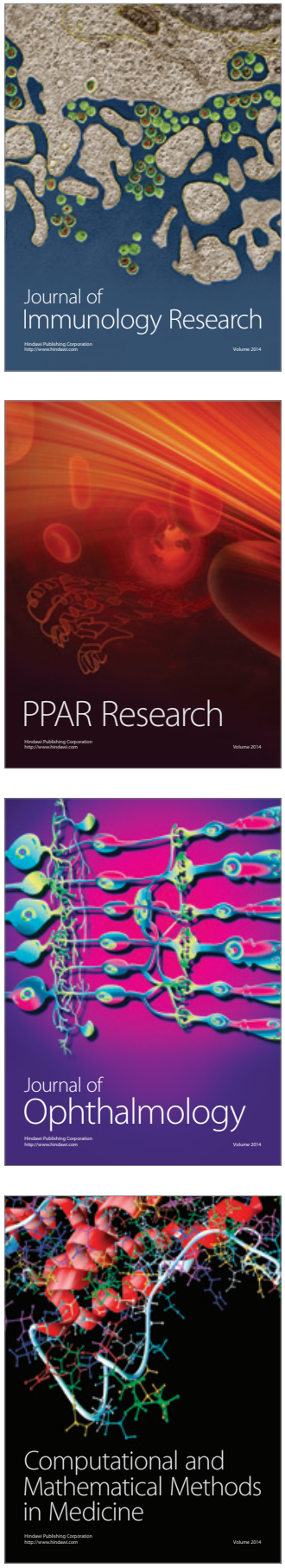

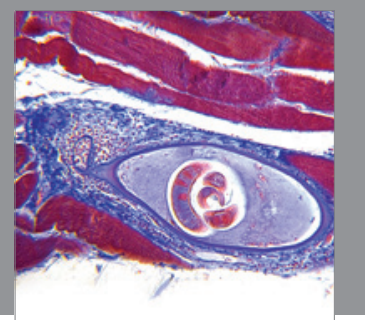

Gastroenterology

Research and Practice
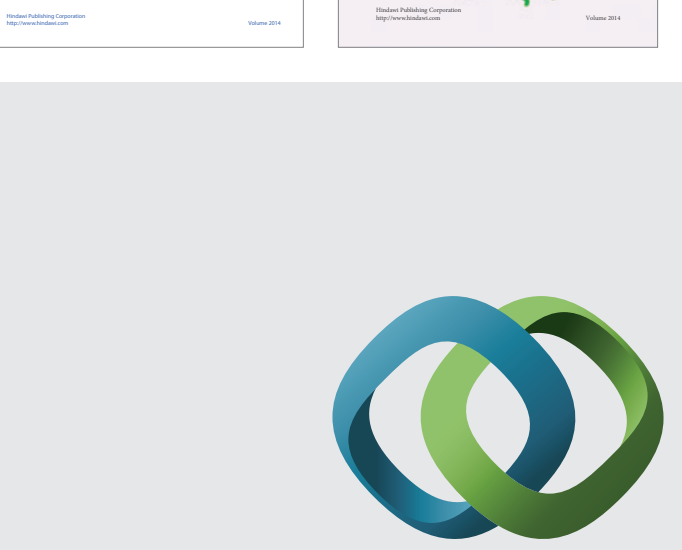

\section{Hindawi}

Submit your manuscripts at

http://www.hindawi.com
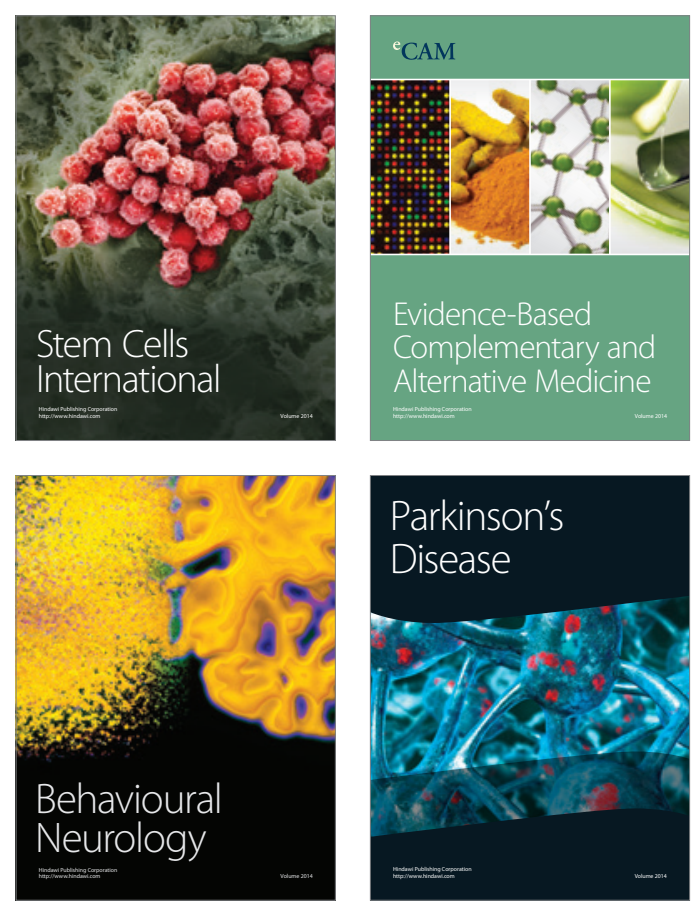

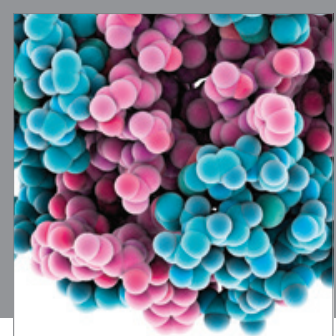

Journal of
Diabetes Research

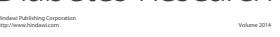

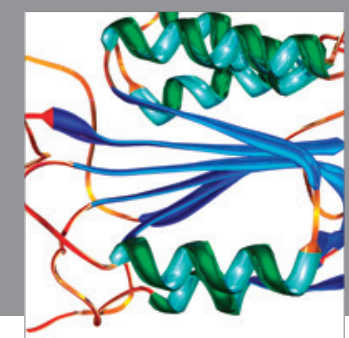

Disease Markers
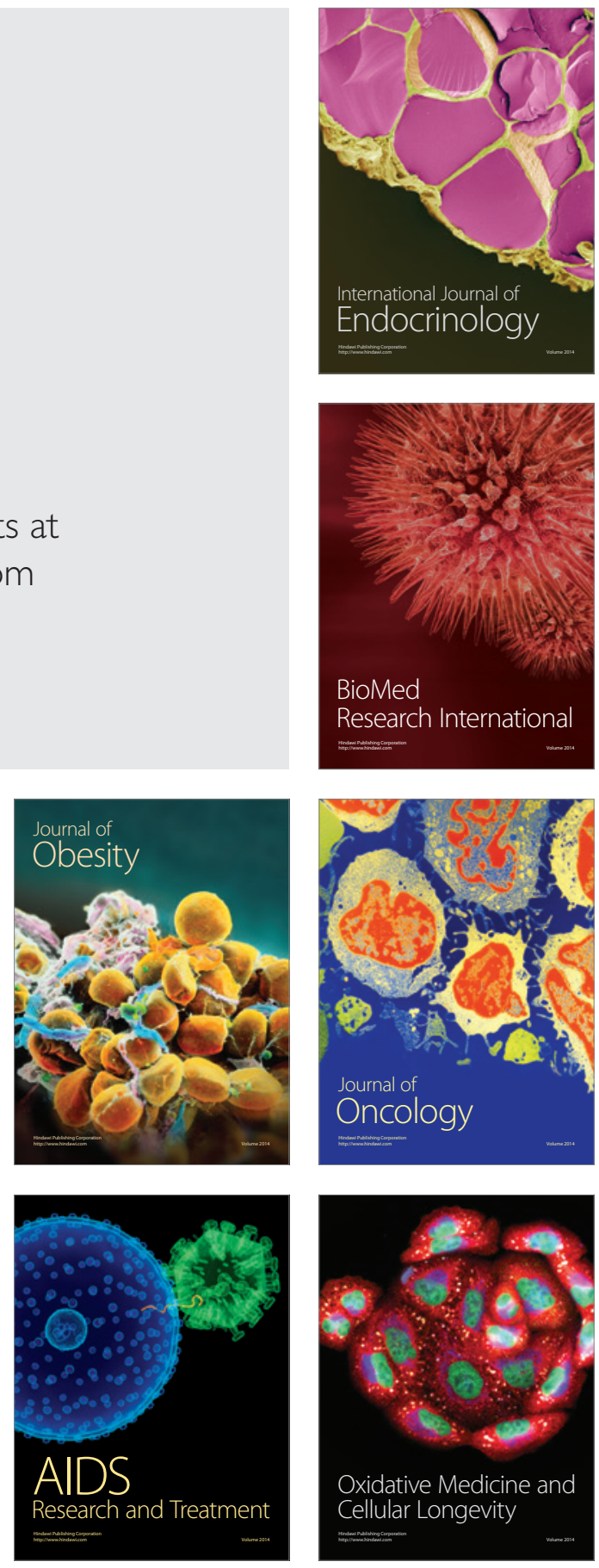